\title{
A QUICK PROOF OF HARISH-CHANDRA'S PLANCHEREL THEOREM FOR SPHERICAL FUNCTIONS ON A SEMISIMPLE LIE GROUP
}

\author{
JONATHAN ROSENBERG ${ }^{1}$
}

\begin{abstract}
Some lemmas of S. Helgason and R. Gangolli, originally conceived for proving an analogue of the Paley-Wiener theorem for symmetric spaces, are used to give a quick proof of Harish-Chandra's inversion formula and Plancherel theorem for bi-invariant functions on a semisimple Lie group. The method is elementary in that it does not require introduction of Harish-Chandra's "Schwartz space."
\end{abstract}

1. Introduction. Among the most beautiful results in harmonic analysis on noncommutative groups are Harish-Chandra's inversion formula and Plancherel theorem for spherical functions on a semisimple Lie group. Unfortunately, however, the original proof of these results requires all of [5] plus some further analysis concerning the "Abel transform" and the " $c$-function," and so is almost inaccessible to the nonexpert. The purpose of this note is to point out how one can shorten the proof of the Plancherel theorem appreciably by using lemmas from [6] and [1] to bypass the difficult analysis in [5], using the main idea of a classical proof of the Plancherel theorem for the real line. The reader of this paper is assumed to be familiar with the results of [4] (also available in [2], [8], and [9, Chapter 9]) and [3], but not with [5]. We also require the main lemmas of [6] and [1] (see [7, pp. 37-39] for a simplication), which will be restated below when we need them.

This paper arose from an attempt to give an elementary exposition of Harish-Chandra's work on spherical functions at a seminar at the University of California, Berkeley, in the spring of 1976. The author would like to thank Jeff Fox for organizing the seminar and getting him interested in the subject, and also Sigurdur Helgason for suggesting some improvements in the original manuscript.

2. Preliminaries and notation. We use the following notation. $G$ is a connected semisimple Lie group with finite center, with Iwasawa decomposition $K A N$, and with identity element $e$. We denote by a the Lie algebra of $A$

Received by the editors August 11, 1976.

AMS (MOS) subject classifications (1970). Primary 22E30, 43A30, 43A90; Secondary 22E45, $33 \mathrm{~A} 75$.

Key words and phrases. Semisimple Lie group, spherical function, inversion formula, Plancherel theorem, $c$-function.

${ }^{1}$ Partially supported by an NSF Graduate Fellowship and by an NSF research grant. 
and by $\mathrm{a}^{*}$ its real dual. We will be working with $\mathscr{K}(K \backslash G / K)$ and with O $(K \backslash G / K)$, the spaces of continuous (resp. $\left.C^{\infty}\right) K$-bi-invariant functions on $G$ with compact support, equipped with the usual inductive limit topologies. (Such functions are determined by their restrictions to $A$.) For convenience, we sometimes identify functions on $A$ with functions on a by means of the exponential map, so that the dual of $\mathscr{D}(K \backslash G / K)$ may be identified with the space of $W$-invariant Schwartz distributions on a, where $W$ is the Weyl group. We denote by $\varphi_{\lambda}$ the elementary spherical function on $G$ corresponding to $\lambda \in a^{*}$, by $c$ the $c$-function on $a^{*}$ which is computed in [3], and by $w$ the order of $W$. The Schwartz spaces of rapidly decreasing functions on the vector spaces $a$ and $a^{*}$ are denoted by $\delta(a)$ and $\delta\left(a^{*}\right)$. Then we have the Euclidean Fourier transform ${ }^{\star}: \delta(a) \rightarrow \delta\left(a^{*}\right)$, and we say Lebesgue measures on $a$ and $a^{*}$ are regularly normalized if this extends to an isometry $L^{2}(a) \rightarrow L^{2}\left(a^{*}\right)$ (i.e., if the Fourier inversion formula holds without a multiplicative constant). Let $\Sigma^{+}$be the set of positive restricted roots for $(G$, $A$ ), let $m_{\alpha}$ denote the multiplicity of a root $\alpha$, and let $\rho=\Sigma_{\alpha \in \Sigma^{+}} m_{\alpha} \alpha / 2$. We let $\mathfrak{a}^{+}$denote the positive Weyl chamber in $a$ and define a function $\Delta$ on $\mathfrak{a}^{+}$ by

$$
\Delta(H)=\prod_{\alpha \in \Sigma^{+}}(2 \sinh \alpha(H))^{m_{\alpha}} .
$$

Then for a suitable choice of Haar measure on $G$, we have

$$
\int_{G} f(x) d x=\int_{a^{+}} f(H) \Delta(H) d H
$$

for all $f \in \mathscr{K}(K \backslash G / K)$. (Here we identify $f$ with $\left(\left.f\right|_{A}\right) \circ$ exp.) For $R>0$, let $B_{R}^{\prime}$ denote the $R$-ball about 0 in a (with respect to the Euclidean metric $|\cdot|$ defined by the Killing form), and call $B_{R}=K \cdot \exp \left(B_{R}^{\prime}\right) \cdot K$ the $R$-ball about $e$ in $G$. For $f \in \mathscr{K}(K \backslash G / K)$, we let

$$
F_{f}(a)=e^{\rho(\log a)} \int_{N} f(a n) d n \quad \text { for } a \in A
$$

(this is the "Abel transform"), where $d n$ is an appropriately normalized Haar measure on $N$, and let

$$
f^{\sim}(\lambda)=\int_{G} f(x) \varphi_{-\lambda}(x) d x \quad \text { for } \lambda \in a^{*}
$$

(this is the "spherical Fourier transform"). Recall from [4, p. 262] (a trivial calculation with the Fubini theorem) that $f^{\sim}=\left(F_{f} \circ \exp \right)^{\wedge}$.

These are the results we need:

Lemma 1 [6, Lemma 5.4]. If $f \in \mathscr{K}(K \backslash G / K)$ has support in $B_{R}$, then $F_{f} \circ \exp$ has support in $B_{R}^{\prime}$. In particular, $F_{f} \circ \exp \in \mathcal{K}(\mathrm{a} / W)$.

LEMMA $2\left(\left[6, p .303\right.\right.$, formula 10]-an immediate consequence of [3]). $|c(\lambda)|^{-2}$ $=O\left(|\lambda|^{\operatorname{dim} N}\right)$ for $|\lambda|$ large. In particular, $|c|^{-2}$ defines a tempered distribution on $a^{*}$. 
Lemma 3 ([6, Proposition 5.1] and [1, Corollary 3.4]). Suppose $\psi \in$ $\mathcal{S}\left(a^{*}\right)$ is the Fourier transform of a W-invariant function in $\mathscr{D}(a)$ with support in $B_{R}^{\prime}$. Then if we define

$$
f(x)=\int_{a^{*}} \psi(\lambda) \varphi_{\lambda}(x)|c(\lambda)|^{-2} d \lambda \quad \text { for } x \in G
$$

(which is meaningful by Lemma 2), $f$ has support in $B_{R}$.

\section{The Theorem and its proof.}

THEOREM (HARISH-CHANDRA). If Lebesgue measure $d \lambda$ on $\mathrm{a}^{*}$ is regularly normalized, then for all $f \in \mathscr{D}(K \backslash G / K)$ and $x \in G$, we have

$$
f(x)=\frac{1}{w} \int_{a^{*}} f^{\sim}(\lambda) \varphi_{\lambda}(x)|c(\lambda)|^{-2} d \lambda
$$

and

$$
\int_{G}|f(x)|^{2} d x=\frac{1}{w} \int_{\mathrm{a}^{*}}\left|f^{\sim}(\lambda) c(\lambda)^{-1}\right|^{2} d \lambda .
$$

Furthermore, the map $f \mapsto f^{\sim}$ extends to an isometry of $L^{2}(K \backslash G / K)$ onto $L^{2}\left(a^{*} / W, w^{-1}|c(\lambda)|^{-2} d \lambda\right)$.

Proof. (a) First we take $x=e$ and consider the map $T$ : $f \mapsto$ $\int_{\mathrm{a}^{*}} f^{-}(\lambda)|c(\lambda)|^{-2} d \lambda$ defined on $\mathscr{D}(K \backslash G / K)$. We observe that this is a distribution (i.e., a linear functional continuous for the topology of $\mathscr{D}$ ). Indeed, $f \mapsto F_{f} \circ$ exp is clearly continuous and linear, and takes $\mathscr{D}(K \backslash G / K)$ into $\mathscr{D}(\mathfrak{a})$ by Lemma 1 . Next ${ }^{\wedge}: \mathscr{D}(\mathfrak{a}) \rightarrow \mathcal{S}\left(a^{*}\right)$ is continuous and linear, while $\int \cdot(\lambda)|c(\lambda)|^{-2} d \lambda$ is a tempered distribution by Lemma 2 . Since $T$ is the composition of all these maps, it, too, is continuous.

(b) Next we show that $T$ has support $\{e\}$. First fix any function $\psi \in \delta\left(a^{*}\right)$ such that $\psi \geqslant 0, \psi(0)=1, \psi$ is radially symmetric, and $\psi$ is of the form $\beta^{\wedge}$ with $\beta \in \mathscr{D}(a)$, say with $\beta$ supported in $B_{1}^{\prime}$. Then for $f \in \mathscr{D}(K \backslash G / K)$,

$$
\begin{aligned}
T f & =\int_{\mathfrak{a}^{*}} f^{\sim}(\lambda)|c(\lambda)|^{-2} d \lambda=\lim _{\varepsilon \downarrow 0} \int_{\mathrm{a}^{*}} f^{\sim}(\lambda) \psi(\varepsilon \lambda)|c(\lambda)|^{-2} d \lambda \\
& \quad \text { (dominated convergence) } \\
& =\lim _{\varepsilon \downarrow 0} \int_{\mathrm{a}^{*}} \int_{G} f(x) \varphi_{-\lambda}(x) d x \psi(\varepsilon \lambda)|c(\lambda)|^{-2} d \lambda \\
& =\lim _{\varepsilon \downarrow 0} \int_{G} f(x) g_{\varepsilon}(x) d x \quad \text { (Fubini), }
\end{aligned}
$$

where

$$
g_{\varepsilon}(x)=\int_{\mathrm{a}^{*}} \varphi_{-\lambda}(x) \psi(\varepsilon \lambda)|c(\lambda)|^{-2} d \lambda=\int_{\mathrm{a}^{*}} \varphi_{\lambda}(x) \psi(\varepsilon \lambda)|c(\lambda)|^{-2} d \lambda
$$

(since $\psi$ and $|c|^{-2}$ are even functions). Thus $T=\lim _{\varepsilon \downarrow 0} g_{\varepsilon}$ in the sense of distributions. But since $\psi=\beta^{\wedge}$ and $\beta$ is supported in $B_{1}^{\prime}$, Lemma 3 shows $g_{\varepsilon}$ is supported in $B_{\varepsilon}$. So $T$ must have support $\{e\}$.

(c) Now we show that $T$ is a measure, which in view of (b) implies that there exists a constant $C$ such that $T f=C f(e)$ for all $f \in \mathscr{D}(K \backslash G / K)$. 
Since $T=\lim _{\varepsilon \downarrow 0} g_{\varepsilon}$, it suffices to prove that $\left\|g_{\varepsilon}\right\|_{L^{\prime}(G)}$ remains bounded as $\varepsilon \downarrow 0$, for then

$$
\begin{aligned}
|T f| & \leqslant \limsup _{\varepsilon \downarrow 0} \int_{G}|f(x)|\left|g_{\varepsilon}(x)\right| d x \\
& \leqslant \limsup _{\varepsilon \downarrow 0}\left\|f f_{B_{\varepsilon}}\right\|_{L^{\infty}}\left\|g_{\varepsilon}\right\|_{L^{\prime}(G)} \leqslant \text { const } \cdot|f(e)| .
\end{aligned}
$$

Now $g_{\varepsilon}$ has support in $B_{\varepsilon}$, so we have

$$
\left\|g_{\varepsilon}\right\|_{L^{1}(G)} \leqslant\left(\text { vol. of } B_{\varepsilon}\right) \cdot\left\|g_{\varepsilon}\right\|_{L^{\infty}} \text {. }
$$

But

$$
\begin{aligned}
\left\|g_{\varepsilon}\right\|_{L^{\infty}} & \leqslant \int_{a^{*}} \psi(\varepsilon \lambda)|c(\lambda)|^{-2} d \lambda=\varepsilon^{-\operatorname{dim} A} \int_{a^{*}} \psi(\lambda)|c(\lambda / \varepsilon)|^{-2} d \lambda \\
& \leqslant \varepsilon^{-\operatorname{dim} A} \int_{a^{*}} \psi(\lambda) \text { const } \cdot(1+|\lambda| / \varepsilon)^{\operatorname{dim} N} d \lambda \quad \text { (by Lemma 2) } \\
& =O\left(\varepsilon^{-\operatorname{dim} A-\operatorname{dim} N}\right),
\end{aligned}
$$

while on the other hand,

$$
\begin{aligned}
\left(\text { vol. of } B_{\varepsilon}\right) & =\int_{\substack{H \in a^{+} \\
|H|<\varepsilon}} \Delta(H) d H \leqslant \text { const } \cdot \int_{\substack{H \in a^{+} \\
|H|<\varepsilon}}|H|^{\operatorname{dim} N} d H \\
& =O\left(\varepsilon^{\operatorname{dim} A+\operatorname{dim} N}\right)
\end{aligned}
$$

for $\varepsilon$ small, since

$$
\Delta(H)=\prod_{\alpha \in \Sigma^{+}}(2 \sinh \alpha(H))^{m_{\alpha}} \approx \text { const } \cdot \prod_{\alpha \in \Sigma^{+}} \alpha(H)^{m_{\alpha}}
$$

for $|H|$ small. Thus $\left\|g_{\varepsilon}\right\|_{L^{\prime}(G)}$ is bounded independent of $\varepsilon$, as desired.

(d) Observe that the statements of the Theorem, aside from the explicit calculation of the constant $C$, follow by means of classical tricks. To get the inversion formula for $f(x)(x \neq e)$, apply the result of step (c) to $g(y)=$ $\int_{K} f(x k y) d k$. To get the formula $C\|f\|_{L^{2}(G)}^{2}=\left\|\tilde{f}^{-1}\right\|_{L^{2}\left(a^{*}\right)}^{2}$, replace $f$ by $f^{*} * f$. One can now deduce the "Paley-Wiener theorem" of [6] and [1] from Lemma 3 and conclude that the spherical Fourier transform maps onto $L^{2}\left(a^{*} / W,|c(\lambda)|^{-2} d \lambda\right)$. (Professor Helgason has kindly suggested the following proof. Suppose $\psi$ and $f$ are as in Lemma 3-we must show that $F=f^{\sim}-\psi$ vanishes identically. Now

$$
\int_{a^{*}} F(\lambda) \varphi_{\lambda}(x)|c(\lambda)|^{-2} d \lambda=0 \quad \text { for all } x \in G,
$$

and integrating against functions in $\mathcal{K}(K \backslash G / K)$, the Fubini theorem gives

$$
\int_{a^{*}} F(\lambda) h^{\sim}(\lambda)|c(\lambda)|^{-2} d \lambda=0 \text { for all } h \in \mathcal{K}(K \backslash G / K) .
$$

Since $\left\{h^{\tilde{L}}: h \in \mathcal{K}(K \backslash G / K)\right\}$ generates the algebra of all continuous functions vanishing at infinity on $a^{*} / W$ (the Stone-Weierstrass hypotheses are satisfied), we get $F(\lambda)|c(\lambda)|^{-2}=0$ a.e., and hence $F \equiv 0$, as desired). 
(e) The last step is to show $C=w$. This is the analogue of the last step in the proof of the Plancherel formula for the line, where one computes with a specific function (usually a Gaussian) to find the correct normalization of Haar measures.

We begin by fixing some $M>0$ and some nonzero $f \in \mathscr{D}(K \backslash G / K)$ whose restriction to $\mathrm{a}^{+}$has support in

$$
\mathfrak{a}^{+}(M)=\left\{H \in \mathfrak{a}^{+} \mid \alpha(H)>M \text { for all } \alpha \in \Sigma^{+}\right\} .
$$

Then for $\varepsilon>0$, we can form a new function $f_{\varepsilon} \in \mathscr{D}(K \backslash G / K)$ with

$$
f_{\varepsilon}(H) \Delta^{1 / 2}(H)=\varepsilon^{(\operatorname{dim} A) / 2} f(\varepsilon H) \Delta^{1 / 2}(\varepsilon H) \quad \text { for } H \in \mathfrak{a}^{+} .
$$

Note that

$$
\left\|f_{\varepsilon}\right\|_{L^{2}(G)}^{2}=\int_{a^{+}}\left|f_{\varepsilon}(H) \Delta^{1 / 2}(H)\right|^{2} d H=\int_{a^{+}}\left|f(H) \Delta^{1 / 2}(H)\right|^{2} d H=\|f\|_{L^{2}(G)}^{2} .
$$

Recall that in the interior of $\mathrm{a}^{+}$, we have the Gangolli expansion [1, equation 3.45]

$$
\Delta^{1 / 2}(H) \varphi_{-\lambda}(H)=\sum_{s \in W} c(-s \lambda) e^{-i s \lambda(H)} \theta_{-s \lambda}(H),
$$

provided that $\lambda \in a^{*}$ is not orthogonal to any root, where

$$
\theta_{-s \lambda}(H)=\sum_{\mu \in L} a_{\mu}(-s \lambda) e^{-\mu(H)}
$$

converges uniformly on compact sets to some analytic function on $a^{+}$. (Here $L$ is the semilattice in $a^{*}$ generated by $\Sigma^{+}$.)

Thus we have for almost all $\lambda \in a^{*}$,

$$
\begin{aligned}
f_{\varepsilon}^{\sim}(\lambda)= & \int_{\mathrm{a}^{+}} f_{\varepsilon}(H) \varphi_{-\lambda}(H) \Delta(H) d H \\
= & \int_{\mathrm{a}^{+}} \varepsilon^{(\operatorname{dim} A) / 2} f(\varepsilon H) \Delta^{1 / 2}(\varepsilon H) \\
& \times \sum_{s \in W} c(-s \lambda) e^{-i s \lambda(H)} \theta_{-s \lambda}(H) d H \\
= & \varepsilon^{-(\operatorname{dim} A) / 2} \int_{\mathrm{a}^{+}} f(H) \Delta^{1 / 2}(H) \\
& \times \sum_{s \in W} c(-s \lambda) e^{-i s \lambda(H) / \varepsilon} \theta_{-s \lambda}(H / \varepsilon) d H .
\end{aligned}
$$

Let $\chi(s, \lambda)=c(-s \lambda) c(-\lambda)^{-1}$ (continuing analytically at poles of $c$ ). Then $\chi$ is continuous on $W \times a^{*}$ with values of modulus one, and

$$
\begin{aligned}
& f_{\varepsilon}^{\tilde{\varepsilon}}(\lambda) c(-\lambda)^{-1} \\
& \quad=\varepsilon^{-(\operatorname{dim} A) / 2} \sum_{s \in W} \chi(s, \lambda) \int_{\mathrm{a}^{+}} f(H) \Delta^{1 / 2}(H) e^{-i s \lambda(H) / \varepsilon} \theta_{-s \lambda}(H / \varepsilon) d H .
\end{aligned}
$$

Let

$$
F_{\varepsilon}(\lambda)=\varepsilon^{-(\operatorname{dim} A) / 2} \sum_{s \in W} \chi(s, \lambda) \int_{a^{+}} f(H) \Delta^{1 / 2}(H) e^{-i s \lambda(H) / \varepsilon} d H
$$


If the Lebesgue measures $d H$ and $d \lambda$ are regularly normalized, dominated convergence and the Plancherel theorem for $a$ and $a^{*}$ show that as $\varepsilon \downarrow 0$,

$$
\begin{aligned}
\frac{1}{w} \int_{a^{*}}\left|F_{\varepsilon}(\lambda)\right|^{2} d \lambda & \rightarrow \frac{1}{w} \int_{a^{*}}\left|\sum_{s \in W} \chi(s, 0) \int_{a^{+}} f(H) \Delta^{1 / 2}(H) e^{-i s \lambda(H)} d H\right|^{2} d \lambda \\
& =\|f\|_{L^{2}(G)}^{2} .
\end{aligned}
$$

Since $C\|f\|_{L^{2}(G)}^{2}=\left\|f_{\varepsilon}^{\tilde{\varepsilon}} c^{-1}\right\|_{L^{2}\left(a^{*}\right)}^{2}$, our desired conclusion $C=w$ will follow if we can show that

$$
\int_{\mathfrak{a}^{*}}\left|f_{\varepsilon}^{\sim}(\lambda) c(-\lambda)^{-1}-F_{\varepsilon}(\lambda)\right|^{2} d \lambda \rightarrow 0 \quad \text { as } \varepsilon \downarrow 0 .
$$

(Recall that $|c(\lambda)|=|c(-\lambda)|$.) Write $f_{\varepsilon}^{\sim}(\lambda) c(-\lambda)^{-1}-F_{\varepsilon}(\lambda)$ in the form $\sum_{s \in W} b_{s}(\varepsilon)$. Since $\left|\sum_{s \in W} b_{s}(\varepsilon)\right|^{2}<w^{2} \sum_{s \in W}\left|b_{s}(\varepsilon)\right|^{2}$, we can treat each value of $s$ separately and estimate as follows:

$$
\begin{gathered}
\int_{\mathrm{a}^{*}}\left|\varepsilon^{-(\operatorname{dim} A) / 2} \chi(s, \lambda) \int_{\mathrm{a}^{+}} f(H) \Delta^{1 / 2}(H) e^{-i s \lambda(H) / \varepsilon}\left[\theta_{-s \lambda}(H / \varepsilon)-1\right] d H\right|^{2} d \lambda \\
=\varepsilon^{-\operatorname{dim} A} \int_{\mathrm{a}^{*}}\left|\int_{\mathrm{a}^{+}} f(H) \Delta^{1 / 2}(H) e^{-i \lambda(H) / \varepsilon}\left[\theta_{-\lambda}(H / \varepsilon)-1\right] d H\right|^{2} d \lambda \\
=\int_{\mathrm{a}^{*}}\left|\int_{\mathrm{a}^{+}} f(H) \Delta^{1 / 2}(H) e^{-i \lambda(H)}\left[\theta_{-\varepsilon \lambda}(H / \varepsilon)-1\right] d H\right|^{2} d \lambda \\
=\left.\int_{\mathrm{a}^{*}} \sum_{\mu \in L-\{0\}} a_{\mu}(-\varepsilon \lambda) \int_{\mathrm{a}^{+}} f(H) \Delta^{1 / 2}(H) e^{-(i \lambda+\mu / \varepsilon)(H)} d H\right|^{2} d \lambda \\
\quad=\int_{\mathrm{a}^{*}} \sum_{\mu, \nu \in L-\{0\}} a_{\mu}(-\varepsilon \lambda) \overline{a_{\nu}(-\varepsilon \lambda)} g(\lambda-i \mu / \varepsilon) \overline{g(\lambda-i \nu / \varepsilon)} d \lambda,
\end{gathered}
$$

where $g$ is the Fourier-Laplace transform of $\left.\left(f \Delta^{1 / 2}\right)\right|_{a^{+}}$. For $\mu \in L$, let $m(\mu)$ denote the level of $\mu$ as defined in [1, p. 158]. Then for $H \in \operatorname{supp} f \subseteq a^{+}(M)$, we have $e^{-(\mu / \varepsilon)(H)}<e^{-m(\mu) M / \varepsilon}$. Using this fact and integrating by parts as in the proof of the classical Paley-Wiener theorem, it is easy to see that for any positive integer $N$ there exists a constant $C_{N}$ such that

$$
|g(\lambda-i \mu / \varepsilon)|<C_{N}(1+|\lambda|)^{-N} e^{-m(\mu) M / \varepsilon} .
$$

Substituting this estimate for a sufficiently large $N$ as well as Gangolli's estimate for the $a_{\mu}(-\varepsilon \lambda)$ [1, formula 3.27] into $(* *)$, we immediately obtain (*).

4. A remark. The expert will note that we have not used Harish-Chandra's "Schwartz space of rapidly decreasing bi-invariant functions," $I(G)$, which occupies a key role in [5]. The reason is that we have tried to make our treatment of the Plancherel theorem as elementary and as self-contained as possible. However, the proof given here also yields the inversion formula for functions in $I(G)$, once one knows (see $\left[9,8.5 .3\right.$ and 9.2.2.3]) that $F_{f} \circ \exp \in$ 
$\delta(a)$ and that $f^{\sim}=\left(F_{f} \circ \exp \right)^{\wedge}$ for $f \in I(G)$. (One merely views $T$ as a tempered distribution in step (a).) But the analysis given here is insufficient to prove that the spherical Fourier transform maps $I(G)$ onto the space of $W$-invariant functions in $\delta\left(a^{*}\right)$. To obtain this, one must first argue as in [5] that if $\psi \in S\left(a^{*}\right)$ is $W$-invariant, then

$$
f(x)=\int_{a^{*}} \psi(\lambda) \varphi_{\lambda}(x)|c(\lambda)|^{-2} d \lambda
$$

defines a function in $I(G)$ which depends continuously on $\psi$. Then the fact that $\psi=f^{\sim}$ follows by continuity from the Paley-Wiener theorem since $\mathscr{D}(a)$ ^ is dense in $\delta\left(a^{*}\right)$.

\section{REFERENCES}

1. R. Gangolli, On the Plancherel formula and the Paley-Wiener theorem for spherical functions on semisimple Lie groups, Ann. of Math. (2) 93 (1971), 150-165. MR 44 \#6912.

2. __ Spherical functions on semisimple Lie groups, Symmetric Spaces, (W. Boothby and G. Weiss, Editors), Dekker, New York, 1972.

3. S. G. Gindikin and F. I. Karpelevič, Plancherel measure for Riemann symmetric spaces of nonpositive curvature, Dokl. Akad. Nauk SSSR 145 (1962), 252-255 = Soviet Math. Dokl. 3 (1962), 962-965. MR 27 \#240.

4. Harish-Chandra, Spherical functions on a semisimple Lie group. I, Amer. J. Math. 80 (1958), 241-310. MR 20 \#925.

5. Spherical functions on a semisimple Lie group. II, Amer. J. Math. 80 (1958), 553-613. MR 21 \#92.

6. S. Helgason, An analogue of the Paley-Wiener theorem for the Fourier transform on certain symmetric spaces, Math. Ann. 165 (1966), 297-308. MR 36 \#6545.

7. __ A duality for symmetric spaces with applications to group representations, Advances in Math. 5 (1970), 1-154. MR 41 \#8587.

8. Analysis on Lie groups and homogeneous spaces, CBMS Regional Conf. Ser. in Math., no. 14, Amer. Math. Soc., Providence, R. I., 1972. MR 47 \# 5179.

9. G. Warner, Harmonic analysis on semisimple Lie groups. II, Springer-Verlag, Berlin and New York, 1972.

Department of Mathematics, University of Pennsylvania, Philadelphia, Pennsylvania 19174 